\title{
Elements Authors Highlight: Nanocartography
}

Matthew Olszta ${ }^{1}$ and Kevin Fiedler ${ }^{2}$

${ }^{1}$ Pacific Northwest National Laboratory, Washington, United States, ${ }^{2}$ United States

Advanced electron microscopy can be extremely challenging yet highly rewarding, with next generation technology allowing us to peer directly into the atomic soul of a material and discern chemical fingerprints at meV resolutions. Yet, even with the highest end microscopes, transmission and scanning transmission electron microscopy (S/TEM) is still regulated by the physical constraints of the sample geometry. Understanding the three dimensional nature of a sample in relation to its crystallographic components, especially at the nanoscale, can mean the difference between average data and achieving highly optimized results. This research proposes a methodology of nanocartography that combines predictive stage motion with crystallographic information to provide microscopists with a sample map that can not only reduce analysis time, but also improve confidence in data collected.(1) Such a road map of stage positions linked to microstructural (e.g., interfaces and growing directions) and crystallographic orientation data (e.g., low index poles and planes) allows unparalleled sample analysis power.

This tutorial will explore the basics of nanocartography, breaking down complex mathematical derivations in order to provide practical materials science analysis in the S/TEM. The authors will demonstrate how to create simple and intuitive crystallographic stage tilt maps that can be combined with the orientation of physical interfaces, such as grain boundaries, that allows full control of sample analysis. Further building upon these basic tenants imbues microscopists with the ability to solve orientation relationships, create oblique tilt series movies, and also solve complex crystallographic unknowns at extremely small length scales with minimal information. Finally, computer coding essentials to translate rigorous mathematical equations and crystallographic mapping concepts will be provided as a means to rapidly orient users as if they were nano-astronauts standing on the sample itself. This introduction to nanocartography will be extremely beneficial to anyone seeking to transform their electron microscopy and take data collection to the next level.

\section{References}

1) Olszta, M., \& Fiedler, K. (2020). Nanocartography in the Age of Automated TEM. Microscopy and Microanalysis, 26(S2), 32-32. 